\title{
Collapse surfaces of the octet-truss lattice at different lattice angles
}

\author{
Mohamed Abdelhamid \\ Department of Mechanical Engineering \\ York University \\ Toronto, Canada \\ mahamid@yorku.ca
}

\author{
Aleksander Czekanski \\ Department of Mechanical Engineering \\ York University \\ Toronto, Canada \\ alex.czekanski@lassonde.yorku.ca
}

\begin{abstract}
Cellular materials are found extensively in nature such as wood, honeycomb, butterfly wings and foam-like structures like trabecular bone and sponge. This class of materials proves to be structurally-efficient by combining low weight with superior mechanical properties. Recent studies have shown there are coupling relations between the mechanical properties of cellular materials and their relative density. Due to its favorable stretching-dominated behavior, continuum models of the octet-truss were developed to describe its effective mechanical properties. However, previous studies were only performed for the cubic symmetry case where the lattice angle $\theta=45^{\circ}$. In this work, we study the impact of the lattice angle on the effective strength of the octet-truss. A graphical method is utilized to plot the collapse surfaces for plastic yielding and elastic buckling for different loading combinations at different lattice angles. Generally, the $\left(\bar{\sigma}_{x z}, \bar{\sigma}_{z z}\right)$ loading space is preferable for lattice angles greater than $45^{\circ}$, while the $\left(\bar{\sigma}_{x x}, \bar{\sigma}_{y y}\right)$ loading space is preferable for lattice angles lower than $45^{\circ}$.
\end{abstract}

Keywords - octet-truss; collapse surfaces; lattice angle; effective strength

\section{INTRODUCTION}

In the last few decades there has been a growing interest in lightweight load-bearing structures. Inspiration from nature can be found in natural cellular materials like wood, honeycomb, butterfly wings, and foam-like structures such as trabecular bones and sponge [1]. Architectural cellular materials have been used to create mechanically-efficient engineering structures such as the Eiffel Tower and the Garabit Viaduct [2]. This class of materials combines the benefits of low density as it only occupies a fraction of the monolithic bulk solid, and strength by arranging its elements efficiently to carry the loads. Previous studies have shown that the macroscopic mechanical properties of cellular materials depend on three parameters: the constituent material properties, the deformation mechanism, and the relative density $\bar{\rho}$ (defined as the solid volume within the unit cell divided by the total volume of the unit cell). Cellular-solids theory predicts scaling relationships between the macroscopic stiffness and strength vs. the relative density, namely $E_{s} \alpha \bar{\rho}^{m}$ and $\sigma_{y} \alpha \bar{\rho}^{n}$ respectively, where the dimensionless parameters $m$ and $n$ depend on the unit cell geometry [3].
For a 3D structure to be rigid (i.e. statically and kinematically determinate), a minimum nodal connectivity of $Z=6$ is required. A connectivity of $Z=12$ categorizes the structure as stretching-dominated where the lattice members deform by tension/compression. Bending-dominated structures that deform through the bending of their members, has a connectivity of $6 \leq$ $Z<12$ [4], [5]. For stretching-dominated structures such as the octet-truss lattice, these scaling relationships are linear. On the other hand, for bending-dominated structures such as honeycombs or the octahedral lattice, they are quadratic or stronger [6], [7].

When the dimensions of the lattice members are scaled down below the micron length scale, they exhibit different mechanical behavior. Examples of these size-dependent changes include strengthening in single crystalline metals and transition from brittle to ductile behavior in metallic glasses and ceramics [8], [9]. Recent advances in additive manufacturing techniques have made it possible to manufacture lattice structures with more geometrical and dimensional freedom. Certain AM techniques like self-propagating photopolymer waveguides [10], projection micro stereolithography [1], and two-photon lithography [11], [12] have been utilized to produce micro and nanolattices within the length scales required to activate material size effects. This is in addition to the structural effects activated by changing the various geometric parameters of the lattice unit cell [13].

Continuum constitutive models have been developed to describe the effective mechanical properties of the octet-truss lattice structure. A common assumption amongst these models is that the lattice members are pin-jointed at all nodes, hence the contribution from the bending resistance of the members and nodes is negligible compared to the axial tensile/compressive stiffness of the members [14]. Deshpande et al. (2001) checked the accuracy of the pin-jointed assumption by comparing FE calculations of rigid-jointed structures against analytical values of pin-jointed models for relative densities $\bar{\rho}$ ranging 0.01 to 0.5 , the results showed excellent agreement between the FE and analytical values proving the validity of this assumption [4]. Generally, symmetry considerations could be employed to deduce the number of independent constants in the macroscopic stiffness tensor. Following the pin-jointed assumption, these elastic constants are determined by averaging the contribution from each element to the macroscopic stiffness, which is achieved through 3D coordinate transformations [15], [16]. 
Nayfeh and Hefzy (1978) derived a first order approximation of the relative density of the octet-truss lattice by dividing the solid volume within the unit cell by the total volume of the unit cell [15]. They employed 3D coordinate transformation and volume averaging in order to obtain the macroscopic stiffness matrix. Lake (1992) constructed a strength tensor by converting applied stresses to strains for each parallel group of members using the macroscopic compliance matrix [17]. Failure would occur in a member if its axial strain exceeded a critical value based on an elastic buckling limit. The choice of elastic buckling over plastic yielding is somehow justified given that space structures, the typical application of lattice structures at that time, usually compose of slender members. Lake's strength tensor could easily accommodate multiaxial loading as well as different loading directions through coordinate transformation. The author also developed a 3D plot of the uniaxial compression strength in cartesian coordinates, from which he concluded the direction and value of the maximum strength of the octet-truss lattice for the case of cubic symmetry where the lattice angle $\theta$ equals $45^{\circ}$ (the angle between the individual members and the horizontal midplane as shown in Fig. 1). Deshpande et al. (2001) investigated the effective properties of the octet-truss lattice structure both theoretically and experimentally [4]. They validated the analytically-predicted elastic modulus and strength using FEM and experimental uniaxial compression of octet-truss lattice made from a casting aluminum alloy. They also explored the collapse criteria of two competing mechanisms (plastic yielding and elastic Euler buckling) and plotted the collapse surfaces for these two mechanisms under different loading conditions. The macroscopic collapse stress was evaluated by equating the external work for the kinematically admissible modes of collapse to: (i) the plastic dissipation in stretching the struts for the case of plastic yielding, (ii) and the internal work in buckling the struts for the case of elastic buckling. In addition, they proposed a third-order approximate formula for the relative density that includes a parameter dependent on the nodes' detailed geometry.

It is important to note that the previous studies were performed only for the case of cubic symmetry. At this angle, the octet-truss is considered to be at the highest attainable level of symmetry. However, potential applications of metamaterials (e.g. thin-walled pressure vessels) necessitates the use of anisotropic lattice structures in order to achieve the optimal combination of low density and high load-carrying capacity.

\section{EFFECTIVE STRENGTH}

The macroscopic strength of the octet-truss lattice is defined as the maximum stress the lattice can sustain without any of its members reaching a critical stress limit. This limit can be defined according to two main modes of failure: (i) yielding for ductile materials or fracture for brittle materials (tensile or compressive), (ii) and Euler beam buckling (compressive). An additional mode of failure for hollow-tube lattices is the shell buckling (compressive). Dominance of either one of these types depends on the loading conditions and the geometry of the lattice members, namely their aspect ratio and cross-section [18].

In order to relate the effective strength of the octet-truss lattice to the local strength of its individual members, the effective macroscopic strains are transformed from the global coordinates to the local member coordinates as follows:

$\varepsilon^{(k)}=N_{i j}^{(k)} \bar{\varepsilon}_{i j}=n_{i}^{(k)} n_{j}^{(k)} \bar{\varepsilon}_{i j}$

where $\varepsilon^{(k)}$ is the axial strain in the $k^{t h}$ member, $N_{i j}^{(k)}$ is a linear transformation operator that can be reduced to the product of the $k^{t h}$ member's direction cosines $n_{i}^{(k)}$ and $n_{j}^{(k)}$, and $\bar{\varepsilon}_{i j}$ is the macroscopic strain, which can be related to a general stress tensor $\left[\bar{\sigma}^{\prime \prime}\right]$ applied through a general direction defined by the angles $\varphi$ and $\alpha$ as shown in Fig. 1 through the following relation:

$$
\left\{\bar{\varepsilon}_{i j}\right\}=[S]\left[T_{1}\right]\left[T_{3}\right]\left\{\bar{\sigma}^{\prime \prime}\right\}
$$

where $[S]$ is the macroscopic compliance matrix, $\left[T_{1}\right]$ and $\left[T_{3}\right]$ are the first and second strain transformation matrices respectively. The reader is referred to [19], [20] for details on the coordinate transformations. By considering the tetrahedron substructure selected as the structural basis in the continuumbased modelling of the octet-truss, the stress in its six members can be expressed as follows:

$\sigma^{(k)}=E_{s} n_{i}^{(k)} n_{j}^{(k)}[S]\left[T_{1}\right]\left[T_{3}\right]\left\{\bar{\sigma}^{\prime \prime}\right\}$

where $i=1,2,3, j=1,2,3$, and $k=1,2 \ldots 6$.

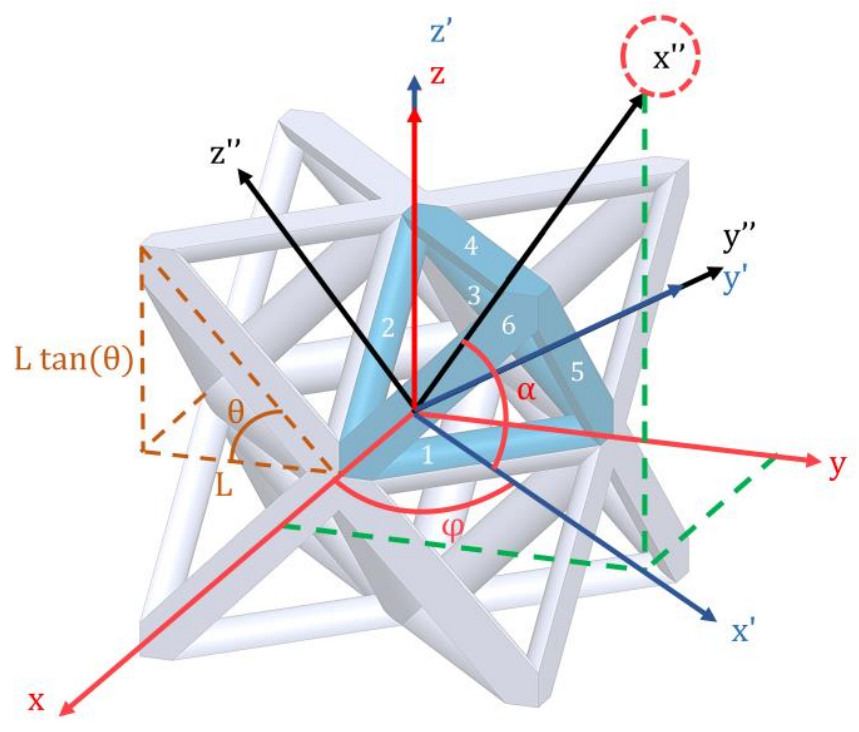

Figure 1. The octet-truss unit cell with the tetrahedron substructure geometry (shown in blue) and the transformation coordinate systems (numbers shown in white identify the six tetrahedron truss members).

\section{COLlaPSE SuRfaces}

The collapse surfaces of the octet-truss lattice due to plastic yielding and elastic buckling are calculated for two combinations of loading, namely $\left(\bar{\sigma}_{x z}, \bar{\sigma}_{z z}\right)$ and $\left(\bar{\sigma}_{x x}, \bar{\sigma}_{y y}\right)$ for different lattice angles. The tetrahedron substructure is used in the analysis in both cases, along with the pin-jointed assumption employed in the continuum-based modelling of the octet-truss. The collapse surface for each combination of loading can be 
categorized into a number of modes depending on the governing collapse equation and which members would reach the stress limit (i.e. buckling or yielding) with reference to Fig. 1. Unlike the analytical methods used by Deshpande et. al (2001) [4], we utilized a graphical method to plot the collapse surfaces. Collapse lines are plotted according to the collapse criteria of each of the six tetrahedron members, the inner-most area is then isolated, and the surrounding collapse lines are used to plot the collapse surface. Hence, fundamentally each of the following governing equation is the collapse criteria of one (or more) of the six tetrahedron members.

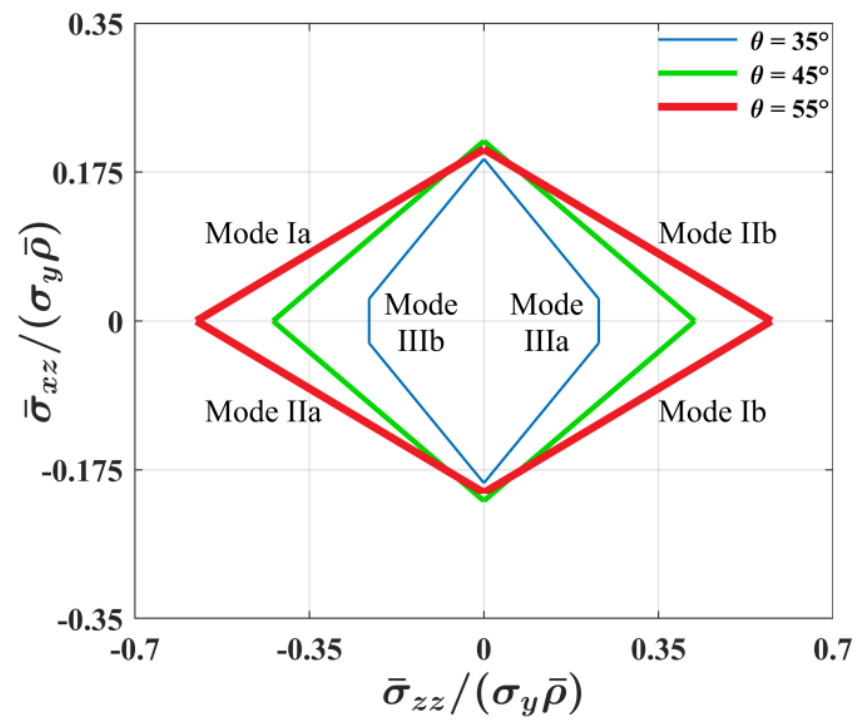

(a)

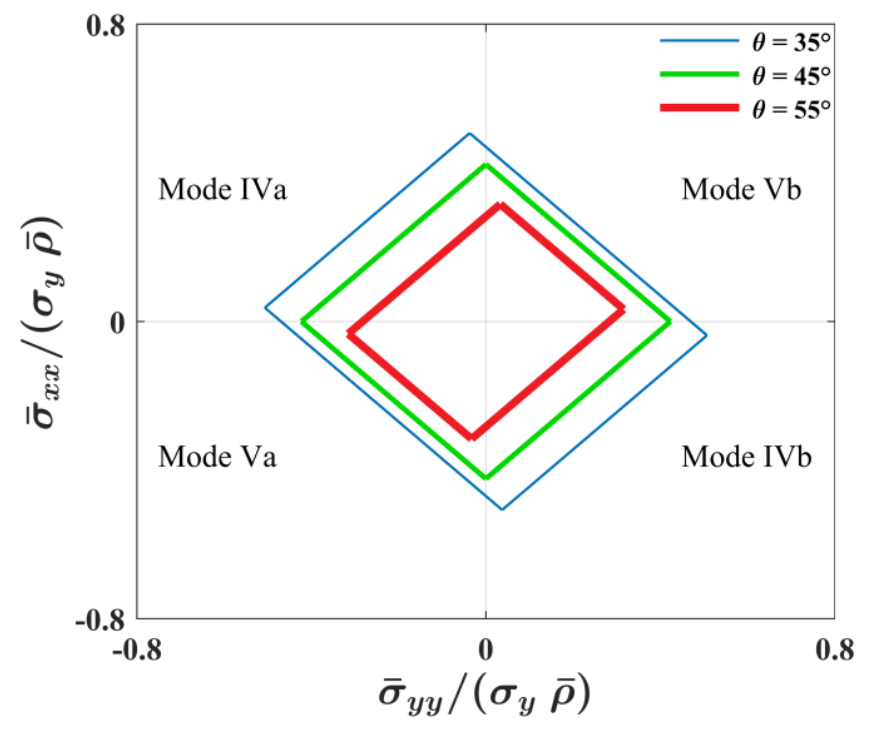

(b)

Figure 2. Collapse surface of the octet-truss due to plastic yielding in $\left(\bar{\sigma}_{x z}, \bar{\sigma}_{z z}\right)$ space (top) and $\left(\bar{\sigma}_{x x}, \bar{\sigma}_{y y}\right)$ space (bottom) in specific strength formulation at an aspect ratio of $r / L=0.14$.
A. $\left(\bar{\sigma}_{x z}, \bar{\sigma}_{z z}\right)$ space due to plastic yielding

The collapse surface under the applied loads $\left(\bar{\sigma}_{x z}, \bar{\sigma}_{z z}\right)$ for three different lattice angles is shown in Fig. 2a using the specific formulation $\bar{\sigma} /\left(\sigma_{\mathrm{y}} \bar{\rho}\right)$.

In Modes $I a$ and $I b$, member no. 2 has reached the yield stress $\sigma_{y}$ (under compression in Mode Ia and tension in Mode $I b)$. The governing equations are as follows:

Mode Ia: $\quad \frac{\bar{\sigma}_{x z}}{\sigma_{y}}=\frac{\bar{\sigma}_{z z}}{2 \tan (\theta) \sigma_{y}}+\cos (\theta) \frac{A_{c}}{L^{2}}$

Mode $I b: \quad \frac{\bar{\sigma}_{x z}}{\sigma_{y}}=\frac{\bar{\sigma}_{z z}}{2 \tan (\theta) \sigma_{y}}-\cos (\theta) \frac{A_{c}}{L^{2}}$

As for Modes II $a$ and $I I b$, member no. 5 has reached the yield stress $\sigma_{y}$ (under compression in Mode IIa and tension in Mode $I I b$ ). The governing equations are as follows:

Mode IIa: $\quad \frac{\bar{\sigma}_{x z}}{\sigma_{y}}=\frac{-\bar{\sigma}_{z z}}{2 \tan (\theta) \sigma_{y}}-\cos (\theta) \frac{A_{c}}{L^{2}}$

Mode $I I b: \quad \frac{\bar{\sigma}_{x z}}{\sigma_{y}}=\frac{-\bar{\sigma}_{z z}}{2 \tan (\theta) \sigma_{y}}+\cos (\theta) \frac{A_{c}}{L^{2}}$

For lattice angles $\theta<45^{\circ}$, there exists two additional Modes, $I I I a$ and $I I I b$, where members no. $1 \& 4$ have reached the yield stress $\sigma_{y}$ (under compression in Mode IIIa and tension in Mode $I I I b)$. The governing equations are as follows:

Mode IIIa: $\frac{\bar{\sigma}_{z z}}{\sigma_{y}}=+\sqrt{2} \tan (\theta) \frac{A_{c}}{L^{2}}$

Mode $I I I b: \quad \frac{\bar{\sigma}_{z z}}{\sigma_{y}}=-\sqrt{2} \tan (\theta) \frac{A_{c}}{L^{2}}$

B. $\left(\bar{\sigma}_{x x}, \bar{\sigma}_{y y}\right)$ space due to plastic yielding

The collapse surface under the applied loads $\left(\bar{\sigma}_{x x}, \bar{\sigma}_{y y}\right)$ for three different lattice angles is shown in Fig. $2 \mathrm{~b}$ using the specific formulation $\bar{\sigma} /\left(\sigma_{y} \bar{\rho}\right)$.

In Modes IVa and IVb, members no. 2, 3,5 \& 6 have reached the yield stress $\sigma_{y}$ (albeit $2 \& 5$ are under tension and $3 \& 6$ are under compression in Mode $I V a$ and the opposite in Mode $I V b)$. The governing equations are as follows:

Mode IVa: $\quad \frac{\bar{\sigma}_{x x}}{\sigma_{y}}=\frac{\bar{\sigma}_{y y}}{\sigma_{y}}-\frac{2 \cos (\theta) A_{c}}{\tan (\theta) L^{2}}$

Mode $I V b: \quad \frac{\bar{\sigma}_{x x}}{\sigma_{y}}=\frac{\bar{\sigma}_{y y}}{\sigma_{y}}+\frac{2 \cos (\theta) A_{c}}{\tan (\theta) L^{2}}$

As for Modes $V a$ and $V b$, members no. $1 \& 4$ have reached the yield stress $\sigma_{y}$ (under compression in Mode $V a$ and under tension in Mode $V b$ ). The governing equations are as follows: 
Mode $V a: \quad \frac{\bar{\sigma}_{x x}}{\sigma_{y}}=\frac{-\bar{\sigma}_{y y}}{\sigma_{y}}-\frac{\sqrt{2} A_{c}}{\tan (\theta) L^{2}}$

Mode $V b: \quad \frac{\bar{\sigma}_{x x}}{\sigma_{y}}=\frac{-\bar{\sigma}_{y y}}{\sigma_{y}}+\frac{\sqrt{2} A_{c}}{\tan (\theta) L^{2}}$

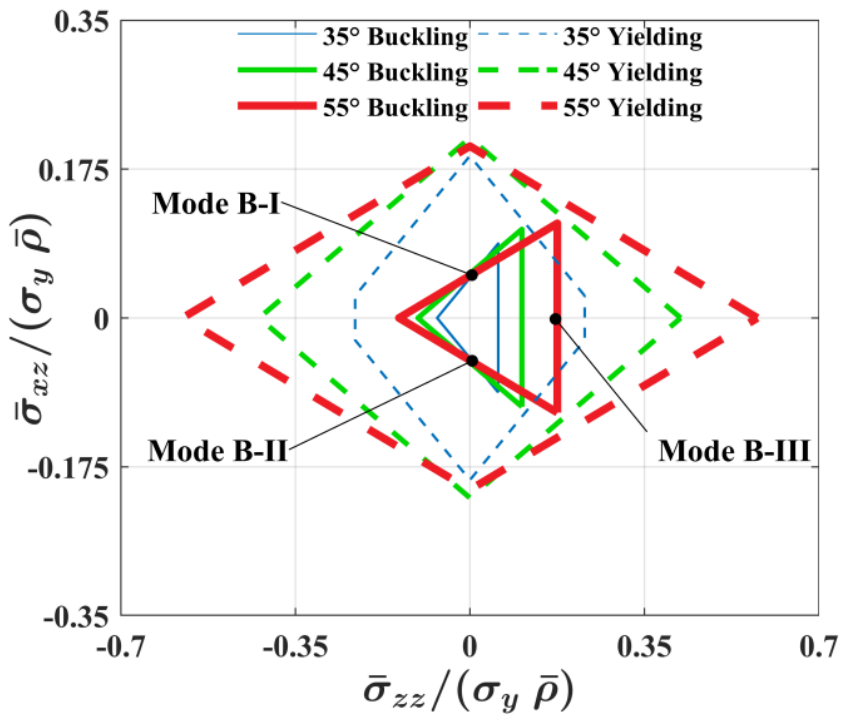

(a)

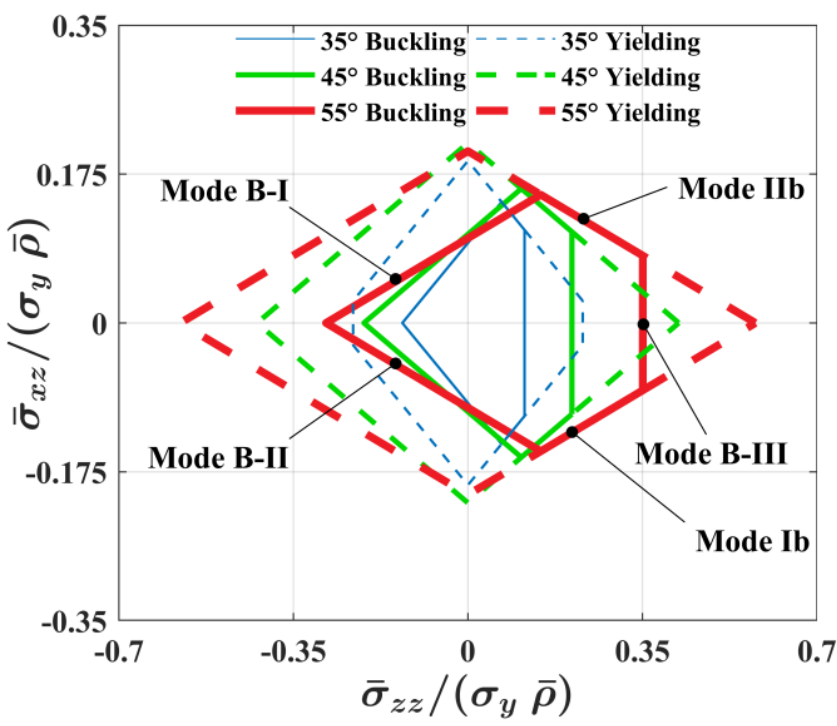

(b)

Figure 3. Collapse surface of the octet-truss due to elastic buckling in $\left(\bar{\sigma}_{x z}, \bar{\sigma}_{z z}\right)$ space at an aspect ratio of $r / L=0.14$ and a yield strain of $\varepsilon_{y}=0.1$ (top) and $\varepsilon_{y}=0.05$ (bottom).

\section{C. $\left(\bar{\sigma}_{x z}, \bar{\sigma}_{z z}\right)$ space due to elastic buckling}

The collapse surface under the applied loads $\left(\bar{\sigma}_{x z}, \bar{\sigma}_{z z}\right)$ for three different lattice angles is shown in Fig. 3 using the specific formulation $\bar{\sigma} /\left(\sigma_{\mathrm{y}} \bar{\rho}\right)$. A yield strain has to be used in order to show both elastic buckling and plastic yielding collapse lines in the same plot. Yield strains of $\varepsilon_{y}=0.1$ and 0.05 are utilized in Fig. $3 \mathrm{a}$ and $3 \mathrm{~b}$ respectively. It can be seen that the yield strain value has no effect on the yielding collapse lines.

In Mode $B-I$, member no. 2 has reached its elastic buckling limit. As for Mode $B-I I$, member no. 5 has reached its elastic buckling limit. In Mode $B-I I I$, members no. $1 \& 4$ have reached their elastic buckling limit. The governing equations are as follows:

Mode $B-I: \quad \frac{\bar{\sigma}_{x z}}{\sigma_{y}}=\frac{+\bar{\sigma}_{z z}}{2 \sigma_{y} \tan (\theta)}+\frac{\pi A_{c}^{2} \cos (\theta)}{8 \varepsilon_{y} L^{4}}$

Mode $B-I I: \quad \frac{\bar{\sigma}_{x z}}{\sigma_{y}}=\frac{-\bar{\sigma}_{z z}}{2 \sigma_{y} \tan (\theta)}-\frac{\pi A_{c}^{2} \cos (\theta)}{8 \varepsilon_{y} L^{4}}$

Mode $B-I I I: \frac{\bar{\sigma}_{z z}}{\sigma_{y}}=\frac{\pi A_{c}^{2} \tan (\theta)}{4 \sqrt{2} \varepsilon_{y} L^{4}}$

\section{D. $\left(\bar{\sigma}_{x x}, \bar{\sigma}_{y y}\right)$ space due to elastic buckling}

The collapse surface under the applied loads $\left(\bar{\sigma}_{x x}, \bar{\sigma}_{y y}\right)$ for three different lattice angles is shown in Fig. 4 using the specific formulation $\bar{\sigma} /\left(\sigma_{y} \bar{\rho}\right)$. Similar to Fig. 3, a yield strain of $\varepsilon_{y}=$ 0.1 and 0.05 are utilized in Fig. $4 \mathrm{a}$ and $4 \mathrm{~b}$ respectively.

In Mode $B-I V$, members no. $2 \& 5$ have reached their elastic buckling limit. As for Mode $B-V$, member no. $3 \& 6$ have reached their elastic buckling limit. In Mode $B-V I$, members no. $1 \& 4$ have reached their elastic buckling limit. The governing equations are as follows:

Mode $B-I V: \frac{\bar{\sigma}_{x x}}{\sigma_{y}}=\frac{+\bar{\sigma}_{y y}}{\sigma_{y}}-\frac{\pi A_{c}^{2} \cos (\theta)}{4 L^{4} \varepsilon_{y} \tan (\theta)}$

Mode $B-V: \quad \frac{\bar{\sigma}_{x x}}{\sigma_{y}}=\frac{+\bar{\sigma}_{y y}}{\sigma_{y}}+\frac{\pi A_{c}^{2} \cos (\theta)}{4 L^{4} \varepsilon_{y} \tan (\theta)}$

Mode $B-V I: \quad \frac{\bar{\sigma}_{x x}}{\sigma_{y}}=\frac{-\bar{\sigma}_{y y}}{\sigma_{y}}-\frac{\pi A_{c}^{2}}{4 \sqrt{2} L^{4} \varepsilon_{y} \tan (\theta)}$

Upon comparison, the collapse surfaces at the lattice angle of $\theta=45^{\circ}$ under the two loading combinations of $\left(\bar{\sigma}_{x z}, \bar{\sigma}_{z z}\right)$ and $\left(\bar{\sigma}_{x x}, \bar{\sigma}_{y y}\right)$ for both plastic yielding and elastic buckling clearly agree with those developed by Deshpande et al. (2001) [4].

\section{CONCLUSIONS}

The purpose of the present research is to investigate the effect of the lattice angle on the effective strength of the octettruss lattice structure. A graphical method is utilized to plot the collapse surfaces for plastic yielding and plastic buckling for different loading conditions and different lattice angles. Generally, the $\left(\bar{\sigma}_{x z}, \bar{\sigma}_{z z}\right)$ loading space is preferable for lattice 
angles greater than $45^{\circ}$, while the $\left(\bar{\sigma}_{x x}, \bar{\sigma}_{y y}\right)$ loading space is preferable for lattice angles lower than $45^{\circ}$.

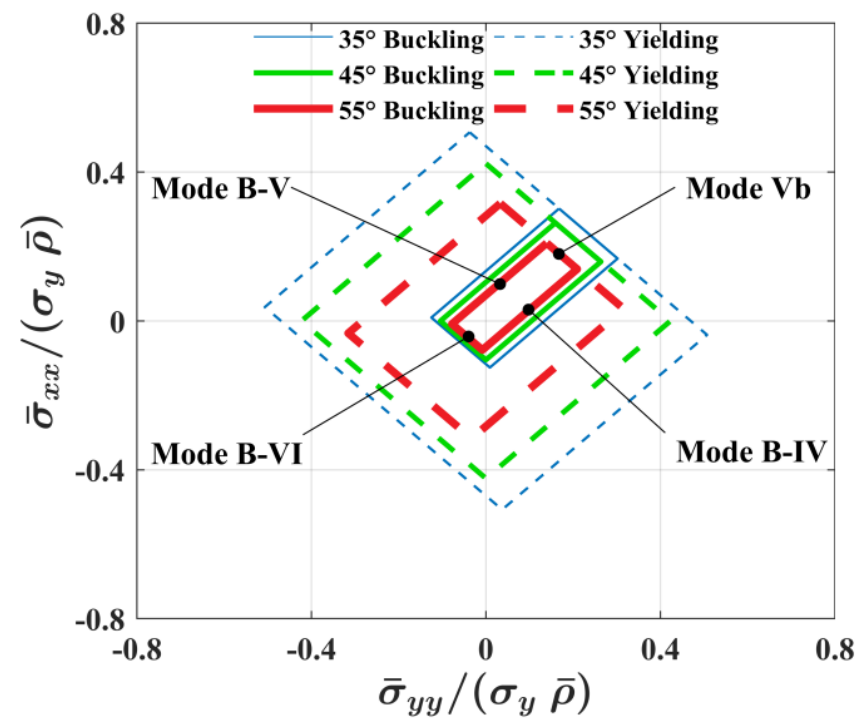

(a)

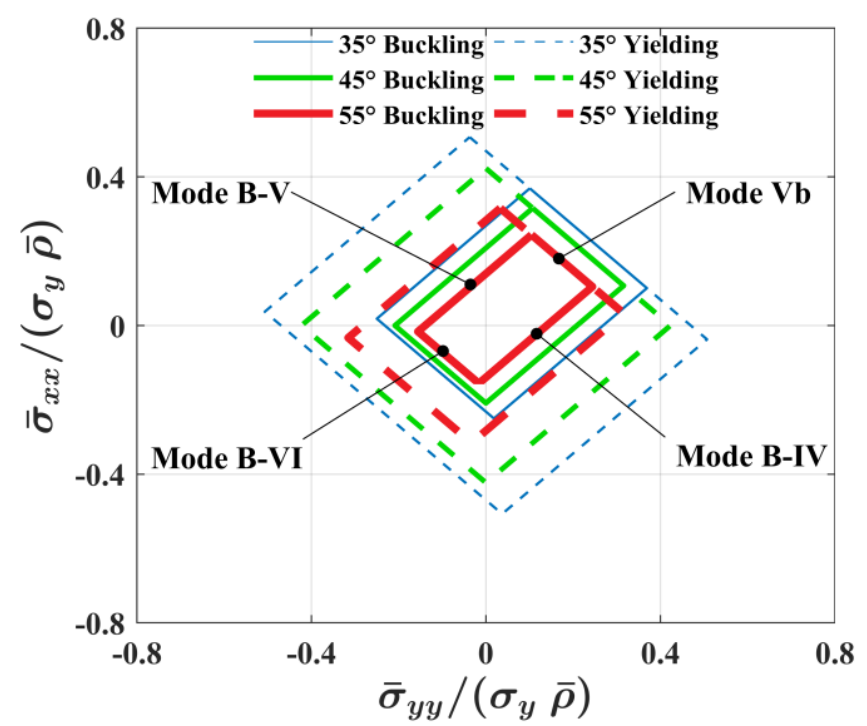

(b)

Figure 4. Collapse surface of the octet-truss due to elastic buckling in $\left(\bar{\sigma}_{x x}, \bar{\sigma}_{y y}\right)$ space at an aspect ratio of $r / L=0.14$ and a yield strain of $\varepsilon_{y}=$ 0.1 (top) and $\varepsilon_{y}=0.05$ (bottom).

\section{REFERENCES}

[1] X. Zheng et al., "Ultralight, ultrastiff mechanical metamaterials.," Science, vol. 344, no. 6190, pp. 1373-7, 2014.

[2] R. Lakes, "Materials with structural hierarchy," Nature, vol. 361, no. 6412, pp. 511-515, Feb. 1993.

[3] L. J. Gibson and M. F. Ashby, Cellular solids: structure and properties, Second. Cambridge: Cambridge University Press, 1997.

[4] V. S. Deshpande, N. A. Fleck, and M. F. Ashby, "Effective properties of the octet-truss lattice material," J. Mech. Phys. Solids, vol. 49, pp. 17471769, Aug. 2001.

[5] V. S. Deshpande, M. F. Ashby, and N. A. Fleck, "Foam topology: bending versus stretching dominated architectures," Acta Mater., vol. 49, no. 6, pp. 1035-1040, 2001.

[6] N. A. Fleck, V. S. Deshpande, and M. F. Ashby, "Micro-architectured materials: past, present and future," Proc. R. Soc. A Math. Phys. Eng. Sci., vol. 466, no. 2121, pp. 2495-2516, Jun. 2010.

[7] M. F. Ashby, T. Evans, N. Fleck, J. W. Hutchinson, H. N. G. Wadley, and L. J. Gibson, Metal foams: a design guide, First. Oxford: Butterworth-Heinemann, 2000.

[8] D. Jang, L. R. Meza, F. Greer, and J. R. Greer, "Fabrication and deformation of three-dimensional hollow ceramic nanostructures.," Nat. Mater., vol. 12, no. 10, pp. 893-8, Oct. 2013.

[9] J. Wang, J. Lian, J. R. Greer, W. D. Nix, and K.-S. Kim, "Size effect in contact compression of nano- and microscale pyramid structures," Acta Mater., vol. 54, no. 15, pp. 3973-3982, 2006.

[10] T. A. Schaedler et al., "Ultralight metallic microlattices," Science, vol. 334, no. 6058, pp. 962-965, Nov. 2011.

[11] L. R. Meza and J. R. Greer, "Mechanical characterization of hollow ceramic nanolattices," J. Mater. Sci., vol. 49, no. 6, pp. 2496-2508, Dec. 2013.

[12] J. Bauer, S. Hengsbach, I. Tesari, R. Schwaiger, and O. Kraft, "Highstrength cellular ceramic composites with 3D microarchitecture.," Proc. Natl. Acad. Sci. U. S. A., vol. 111, no. 7, pp. 2453-8, Feb. 2014.

[13] L. C. Montemayor and J. R. Greer, "Mechanical response of hollow metallic nanolattices: combining structural and material size effects," $J$. Appl. Mech., vol. 82, no. July, pp. 1-10, 2015.

[14] J. Mikulas, M. M., H. G. Bush, and M. F. Card, "Structural stiffness, strength and dynamic characteristics of large tetrahedral space truss structures," 1977.

[15] A. H. Nayfeh and M. S. Hefzy, "Continuum modeling of threedimensional truss-like space structures," AIAA J., vol. 16, no. 8, pp. 779-787, Aug. 1978.

[16] K. Heki, "On the effective rigidities of lattice plates," Recent Res. Struct. Mech., pp. 31-46, 1968.

[17] M. S. Lake, "Stiffness and strength tailoring in uniform space-filling truss structures," Nasa Tech. Pap. 3210, pp. 1-28, Apr. 1992.

[18] L. R. Meza, S. Das, and J. R. Greer, "Strong, lightweight, and recoverable three-dimensional ceramic nanolattices," Science, vol. 345, no. 6202, pp. 1322-1326, Sep. 2014.

[19] M. Abdelhamid and A. Czekanski, "On the effective properties of 3D metamaterials," in ASME, Volume 9: Mechanics of Solids, Structures and Fluids; NDE, Diagnosis, and Prognosis, 2016, p. V009T12A062.

[20] M. Abdelhamid and A. Czekanski, "Impact of the Lattice Angle on The Effective Properties of The Octet-truss Lattice Structure," J. Eng. Mater. Technol., 2018 (accepted). 